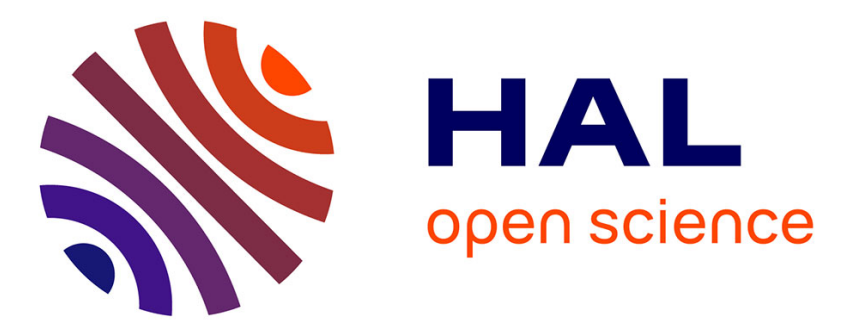

\title{
Submicronic Raman and transverse dynamic force microscopy spectroscopies to investigate the physical chemistry of surface nanoheterogeneities
}

B. Humbert, J. Grausem, Daniel Courjon

\section{- To cite this version:}

B. Humbert, J. Grausem, Daniel Courjon. Submicronic Raman and transverse dynamic force microscopy spectroscopies to investigate the physical chemistry of surface nanoheterogeneities. Journal of Physical Chemistry B, 2004, 108 (40), pp.15714-15720. 10.1021/jp036570d . hal-00096923

\section{HAL Id: hal-00096923 \\ https://hal.science/hal-00096923}

Submitted on 13 May 2021

HAL is a multi-disciplinary open access archive for the deposit and dissemination of scientific research documents, whether they are published or not. The documents may come from teaching and research institutions in France or abroad, or from public or private research centers.
L'archive ouverte pluridisciplinaire HAL, est destinée au dépôt et à la diffusion de documents scientifiques de niveau recherche, publiés ou non, émanant des établissements d'enseignement et de recherche français ou étrangers, des laboratoires publics ou privés. 


\title{
Submicronic Raman and Transverse Dynamic Force Microscopy Spectroscopies to Investigate the Physical Chemistry of Surface Nanoheterogeneities
}

\author{
Bernard Humbert, ${ }^{* \dagger}{ }^{\dagger}$ Jérôme Grausem, ${ }^{\dagger}$ and Daniel Courjon ${ }^{*}$ \\ Laboratoire de Chimie Physique et Microbiologie pour l'Environnement, UHP Nancy I-CNRS, UMR 7564, \\ 405 rue de Vandoeuvre, 54600 Villers-lès-Nancy, France, and Laboratoire d'Optique P.M. Duffieux, \\ Université de Franche-Comté-CNRS, UMR 6603, route de Gray, 25030 Besançon Cedex, France
}

On the basis of an experimental example, this paper demonstrates the intrinsic complementary nature between the subwavelength Raman spectroscopy and the tip-sample interaction measurements, in a scanning near field optical microscope device. This paper displays a good illustration of possibilities given by this kind of device to study the interfaces in physical chemistry. The Raman spectra of the optical near field obtained on a lead sulfate sample shows that the surface of this sample is polluted by nanophases of copper sulfate. These impurities, which are not displayed by the usual backscattered far field Raman spectrum recorded in confocal Raman microspectroscopy, are localized at a subwavelength scale and are revealed by a particular behavior in transverse dynamic force (usually noted as shear force) measurements too, in comparison with those obtained over the surface of the lead sulfate sample free from impurities. These differences in probesample adhesion throughout a sample are caused by an inhomogeneous distribution of surface water, which is clearly explained by the chemical identification of the nanophases by Raman spectroscopy of the optical near field. The heterogeneous water adsorption at the sample/air interface under a reduced relative humidity is displayed by both the near field optical spectroscopy and the shear force microscopy measurements.

\section{Introduction}

Predicting how solids grow and dissolve in aqueous medium under various conditions requires a thorough understanding of the fundamental surface reaction mechanisms. Surface heterogeneities of either compositional or morphological origin are known to drastically affect interface phenomena. Depending on their chemical and topological pattern, these surface heterogeneities often generate a wide variety of singularities when they are involved in interfacial phenomena. Scanning probe microscopy (SPM) may be used to investigate the superficial mechanisms that control dissolution, precipitation, or growth of minerals. Sulfate anions are often cited in such mechanisms in environmental studies. Sulfate strongly adsorbs to many minerals surfaces and competes with other adsorbing compounds for surface sites. Questions about the mechanism of sulfate adsorption and the structure of sulfate surface complexes remain. For example, the following issues are not resolved:

(i) is sulfate adsorbed as an innersphere or an outersphere complex? (Inner sphere in this context refers to the situation where sulfate replaces one or several surface hydroxyl groups, resulting in the coordination of at least one sulfate oxygen atom with a surface metal site.)

(ii) Is the structure mono or bidentate?

(iii) Is the structure mono or binuclear, chelating or bridging?

(iv) Is the spatial distribution of the adsorption sites homogeneous at the interface or is it heterogeneous?

To answer this nonexhaustive listing of questions, one must use an experimental approach able to combine both localization and structural-chemical identification. For example, the surface complexation of sulfate by hematite has been studied by

* Corresponding author. E-mail: humbert@1cpe.cnrs-nancy.fr.

† Laboratoire de Chimie Physique pour l'Environnement.

Université de Franche-Comté-CNRS. vibrationnal infrared absorption spectroscopy and scanning tunneling microscopy on the same sample by Eggleston et al., ${ }^{1,2}$ However, in their work, both experiments are carried out separately, one after another. Therefore, we have developed an experimental method combining submicronic localization and Raman spectroscopy. ${ }^{3,4-7}$ Indeed, Jancke et al. demonstrated the feasibility of the Raman spectroscopy of the optical near field with a lateral resolution smaller than $100 \mathrm{~nm} .{ }^{8,9}$ In this paper, we report our experiments of near field Raman spectroscopy (NFRS) obtained without exaltation effect on metallic sulfate samples. Initially, our experimental goal was to know if the sulfate groups may be a molecular probe that is active enough in NFRS experiments to localize and characterize submicronic spatial heterogeneous areas. Consequently, a first step was to show that the NFRS was able to separate two kinds of sulfate complexes. This experimental step drove us to consider some synthetic samples with a controlled mixture of several metallic sulfate phases. These mixtures display experimentally also a large difference in their tip-sample adhesion behaviors. Indeed, our optical device using a distance control mechanism that measures the amplitude of the interactions between the optical tip and the sample has shown that the vibrating behavior of the tip differs from a sulfate sample to another. In this paper, we consider one of our samples that has been accidentally obtained but that illustrated the complementarities of the NFRS and of the force microscopy.

For a long time, diffraction inherent in all magnifying lenses has limited the resolution of classical optical microscope to onehalf of the wavelength of the light (typically $250 \mathrm{~nm}$ for visible light). In 1928, E. H. Synge proposed a novel optical microscope that could overcome the diffraction barrier. ${ }^{10,11}$ In 1982, most of the mechanical problems were solved by the invention of the scanning tunneling microscope (STM), ${ }^{12}$ and the first 
scanning near field optical microscope (SNOM or NSOM) had been demonstrated in 1984 by D. Pohl. ${ }^{13}$ At present, SNOM is often combined with shear force feedback to maintain the tip to sample distance and uses high optical throughput fiber optic etched probes. ${ }^{14,15}$ Super resolved transmission or reflection images providing both topographical and optical properties (i.e., local refractive index) are obtained now commonly with a resolution of $\lambda / 10$. Resolutions of $\lambda / 20$ have been achieved. ${ }^{16}$

One of the most powerful applications of SNOM should be subwavelength spectroscopy, providing spectral contrasts at subwavelength resolution. An ambitious application was to develop a Raman near field microscope. Rather than obtaining a better spatial lateral resolution than those obtained with a confocal far field device, one of the aims in developing NFRS would be the spectroscopic study of submicronic heterogeneities at the interfaces. Indeed, since the near field decreases strongly versus the distance, the optical near field in the solid phase collected by a nanodetector or generated by a nanosource is dominated by the signals coming from the volume close to the interface (signals coming from a depth beyond $100 \mathrm{~nm}$ are four times weaker than the ones coming from the surface). ${ }^{7,17}$

With both the developments of lasers and detectors (CCD), but also with the elaboration of high throughput probes with research concerning the geometry of the tip, the inelastic Raman effect, even with its poor quantum efficiency, is detectable in the near field..$^{3-7}$ Recently, NFRS has been used to study silicon, polydiacetylene, diamond, Rd-doped $\mathrm{KTP}$, rhodamine $6 \mathrm{G}$, 3-hydroxykynurenine, cobalt phthalocyanine, benzoic acid, $\mathrm{CCl}_{4}$, polyphenylenevinylene, and $\mathrm{CuSO}_{4} \cdot 5 \mathrm{H}_{2} \mathrm{O} \cdot .^{3-9,17-34}$ In our previous works, the Raman spectra of the $\mathrm{CuSO}_{4}$ sample were collected in the optical near field, without assigning the spectral differences between near field and far field spectra. ${ }^{6}$ In this work, we have driven an experiment on a $\mathrm{PbSO}_{4}$ sample under a relative humidity of $5-10 \%$ at $24{ }^{\circ} \mathrm{C}$. We will discuss the recorded NFR spectra in relation to the local water adsorption displayed by the tip-sample shear force measurements.

\section{Experimental Procedures}

The Raman excitation source is a Spectra-Physics laser Stabilite 2017 (wavelength used: $\lambda=514.5 \mathrm{~nm}$ ). The spectrometer is a Jobin-Yvon Raman T64000, with a liquid nitrogen cooled CCD multichannel detector. A holographic NOTCH filter or a double subtractive stage with 1800 grooves $\mathrm{mm}^{-1}$ gratings may be used to remove the Rayleigh scattering. A single dispersion stage with an 1800 grooves $\mathrm{mm}^{-1}$ grating is placed in front of the detector. The spectral resolution with an excitation at $514.5 \mathrm{~nm}$ is $2.7 \mathrm{~cm}^{-1}$. The confocal Raman microprobe is constituted by an Olympus microscope, equipped with a motorized $X Y$ stage with a step of $80 \mathrm{~nm}$. The lateral Raman resolution has been estimated on a sample built with silicon lines of $3 \mu \mathrm{m}$ in a width separated by $3 \mu \mathrm{m}$ on a gold substrate. ${ }^{7}$ To estimate the spatial resolution, we will consider the lateral resolution definition as the minimal distance to separate two different points of a test sample that is constituted by different chemical species. However, it is very difficult to prepare such samples at scales of $100 \mathrm{~nm}$. Therefore, we estimate the lateral resolution only at the boundaries of an object: the lateral resolution value will be the necessary displacement between maximum to minimum collected signal. ${ }^{7}$ This definition may be, of course, questionable, since for instance, it depends on the signal/noise ratio, but in practice for our experiments at the subwavelength scales, it is the only definition that can be applied. To be coherent in this paper, we have applied this definition to estimate the resolution of our conventional confocal device: the measured value is $700 \mathrm{~nm}$.

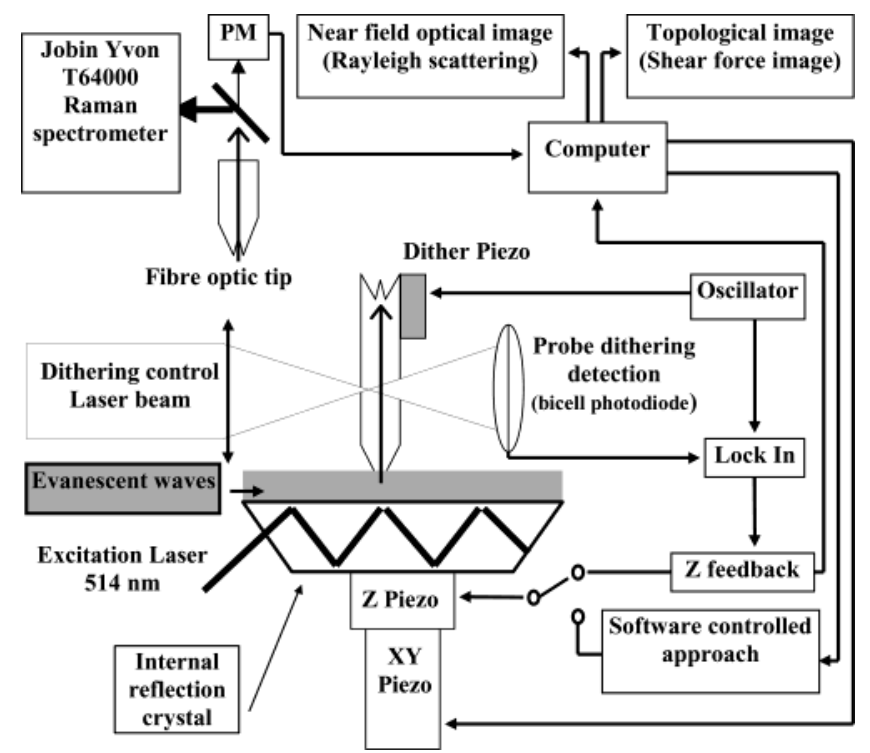

Figure 1. Schematic of the experimental configuration. The evanescent waves generated by an internal reflection of the exciting laser are frustrated by the probe, which is kept close to the surface by a feedback loop that measures the damping of the lateral vibration amplitude of the tip. A small laser beam is focused before the fiber tip, and the modulation of its shadow is detected on the bicell photodiode. This signal, which is strongly damped near the surface, gives a measure for the tip-sample interaction and is used as a reference for the feedback loop that controls the vertical position of the sample via the piezoelectric ceramic scanner $(Z)$. The signals collected by the tip are driven to a beam splitter, which transmits about $25 \%$ of the whole intensity on a photomultiplier and reflects $75 \%$ on the entrance slit of the spectrometer T64000. Thus, by collecting the $Z$ values vs position $(x, y)$, a topographic image is constituted, and by collecting the intensity of the $\mathrm{PM}$, an optical image is obtained.

The homemade near field microscope is schemed in Figure 1. Its principle is based on a shear force scanning near field optical microscope developed previously by Courjon et al. (a review on the usual near field optics and their applications is given in the book of M. A. Paesler et al.). ${ }^{35,36}$ To simplify Figure 1 , the optical near field is schematically obtained by a total internal reflection. For our samples, the near field is generated by an oblique far field illumination and collected by the tip. This mode, rarely used in the literature, will be justified by our experimental results.

As for other experiments in optical near field, the fiberoptic probe can be used in three different ways to record a near field Raman spectrum:

(i) as a nanolight source,

(ii) as a detector that frustrates the evanescent waves in total internal reflection experiments or that is polarized by an optical near-field, or

(iii) as both nanolight source and detector; in this configuration, the tip produces the evanescent waves that are collected by the same aperture for reflection experiments, a method that should give the best spatial resolution. ${ }^{37}$

In NFRS, one of the most important problems is the strong silica Raman spectrum of the fiberoptic or glass probe when used as both emitter and collector. This spectrum covers all other Raman signals in a range of $0-1300 \mathrm{~cm}^{-1}$. The only solution consists of using the probe only to excite (i) or to collect (ii) the Raman spectrum. Jahncke et al. have reported the first near field Raman images, using the probe as a nanolight source. ${ }^{8,9}$ But today, the small Raman signal levels and the long integration times, often several hours for one image, limit the imaging possibilities of the method. Most of the time, a single 


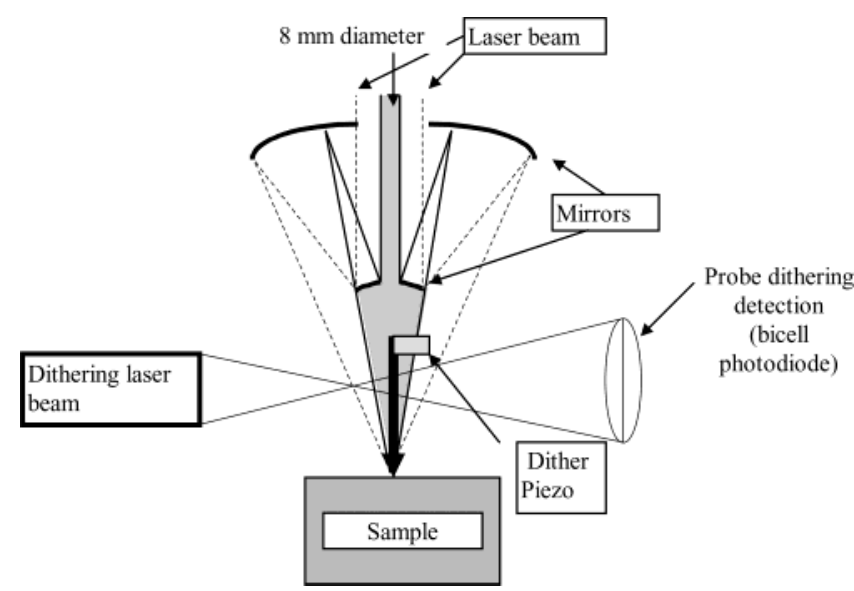

Figure 2. Schematic diagram of the experimental setup to obtain both far and near fields of the same spatial range.

dimensional transverse measurement is possible. ${ }^{5,7,17}$ Fortunately, besides the imaging of interfaces, the NFRS or the near field fluorescence spectroscopy with high spectral resolution is able to reach the detection and the study of single molecules. A classical SNOM experience gives the topography of the sample and locates an optical or topographical heterogeneity. Once localized, the near field Raman spectrum of a nanodomain can be obtained with minimal perturbations, without averaging their physical properties in the space and without masking them by a statistical distribution, which is the case in usual propagated far field experiments.

Figure 2 illustrates the device used here to collect simultaneously both far and near inelastic fields of one spatial range. A far field optical device allows us to focus a laser beam on an area of about $100 \mu \mathrm{m}^{2}$ around the tip. This device is based on a Cassegrain objective (Tri-star, Jobin-Yvon) working above the near field probe (Figure 2). The lateral resolution of the far field optical device equipped with this Cassegrain objective is about $10 \mu \mathrm{m}$, while the axial resolution increases to $20 \mu \mathrm{m}$. The distance feedback loop in our scanning near field optical microscopy device is based on the measurement of the forces perpendicular to the tip-sample axis. Thus, our device gives a transverse dynamic force image (TDFM). ${ }^{38}$ We prefer this last definition rather than the usual shear force expression used in the literature in the past few years. Indeed, the term shear force is often not appropriate because the interactions between surfaces are not in all cases shear. The near field probe and the piezoelectric transducer exciting the transverse-force vibration are positioned in the cone of shadow of the far field objective. This far field objective allows us to collect also the backscattered far fields.

The near field probe is either a Topometrix metallic single mode fiber optic, developed for the SNOM Aurora of Topometrix, with an aperture between 50 and $100 \mathrm{~nm}$ in diameter, or a homemade taper obtained by the commonly used heating and pulling technique. This later probe is built with a commercial instrument used for micropipet manufacture. An optical fiber is placed under tensile stress and locally heated with a $\mathrm{CO}_{2}$ laser. When softening is sensed, the tension is increased, and the laser beam is stopped. The subsequent fracture results in a taper with a flat cleave at the end of about $50 \mathrm{~nm}$ in diameter. The taper is then made metallic by evaporation of aluminum in a vacuum chamber. The other end of the fiber is coupled to the spectrometer's microscope through a $\times 50$ objective, with a 0.55 numerical aperture. Figure 3 gives some electronic microscope images obtained on the tapers used in this work. The choice of the tip in our NFRS experiments is related to its ability to
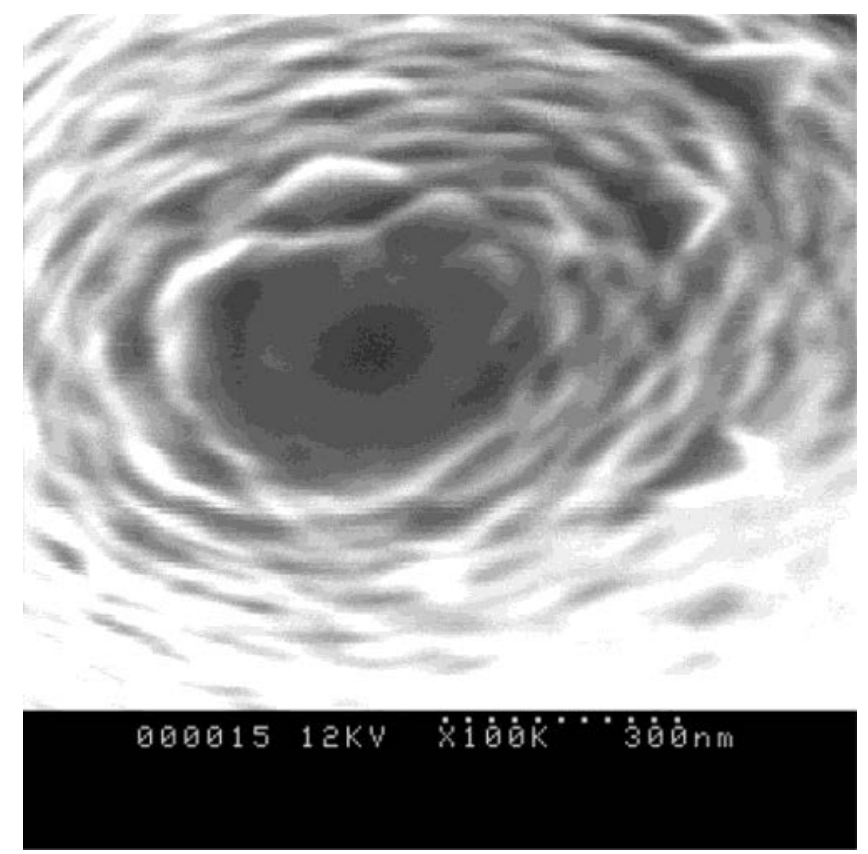

Figure 3. Electronic microscope image of an Al-coated taper. The black center corresponds to the silica aperture. The aperture diameter is estimated at $150 \mathrm{~nm}$ and the aluminum coating at $210 \mathrm{~nm}$.

frustrate an evanescent wave with a minimum of transmitted far field. Indeed, the collection of far field by the tip may represent a strong handicap for the extremely weak near field Raman spectrum as illustrated in the following results. The homogeneity as the thickness of the aluminum coated film but also the size of the aperture at the taper end are the essential parameters to minimize the collection of the far fields. Figure 3 displays the quality of the taper ends. But this visual quality of the end is not sufficient to guarantee the quality of the film in the superior part of the taper and eventually the degradation of the tip during experiments. Therefore, we check empirically the near field character of our tip by collecting the Rayleigh signal in a sample-tip retract experiment. A tip is considered being usable if, when the tip is separated continuously from optical near field region (damping of the shear force signal of $50 \%$ ) to about $1 \mu \mathrm{m}$ of the sample, the optical elastic signal is divided by a factor of about 30 .

\section{Results}

Figure 4 displays the far field backscattered Raman spectrum of the sulfate lead sample studied here. This spectrum may be assigned either to the $\mathrm{PbSO}_{4}$ isomorphous to the $\mathrm{BaSO}_{4}$ or to the lead sulfate tribasic $3 \mathrm{PbO} \cdot \mathrm{PbSO}_{4}, x \mathrm{H}_{2} \mathrm{O}$. However, the chemical analyses and a systematic polarized Raman study prove that the sample is a pure phase of $\mathrm{PbSO}_{4}$ crystallized in the orthorhombic system. The space group is $V_{h},{ }^{16}$ with four $\mathrm{SO}_{4}{ }^{2-}$ ions and four $\mathrm{Pb}^{2+}$ ions per unit cell. The site symmetries of these ions is $C_{3}$. Each sulfate ion is surrounded by seven lead ions, three of these being in the same plane as the sulfur atom. The isolated sulfate ion, in the point group $T_{d}$, possesses only four different levels, noted usually $v 1$ for the nondegenerated symmetric stretching vibration, $v 2$ the doubly degenerated deformation $E, v 3$ the triply degenerated stretching vibration $T_{2}$, and $v 4$ the triply degenerated bending mode $T_{2}$. In the crystals, each sulfate ion occupies a site of $C_{3}$ symmetry, and the anisotropic crystal field results in the complete lifting of the sulfate degenerated modes. The $v 1$ mode gives then in the solid an $A_{1 g}$, a $B_{2 g}$, and $B_{3 g}$ modes, all at $977 \mathrm{~cm}^{-1}$. The $v 3$ 


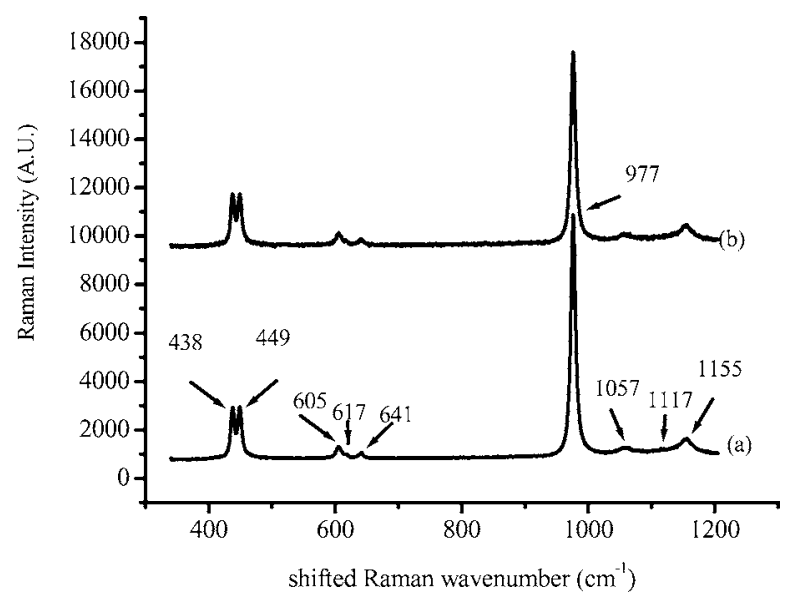

Figure 4. Raman spectrum of the $\mathrm{PbSO}_{4}$ sample, excited at $514 \mathrm{~nm}$ with a power of $5 \mathrm{~mW}$ focused on the sample with an $\times 50$ objective (numerical aperture of 0.5), spectrum a and with the Cassegrain objective $\left(20 \mathrm{~mW}\right.$ on about $\left.100 \mu \mathrm{m}^{2}\right)$, spectrum $\mathrm{b}$. The relative intensities are weakly different between both spectra because of the difference of the instrumental polarization responses between both objectives.

mode gives two $A_{1 g}$ modes, two $B_{2 g}$ modes, and one $B_{3 g}$ mode, respectively, at $1060,115,1054,1157$, and $1152 \mathrm{~cm}^{-1}$. The $v 4$ gives two $A_{1 g}$ modes, two $B_{2 g}$ modes, and one $B_{3 g}$ modes, respectively, at $605,618,610,641$, and $609 \mathrm{~cm}^{-1}$. At last, the $v 2$ gives one $A_{1 g}$ mode, one $B_{1 g}$ mode, one $B_{2 g}$ mode, and one $B_{3 g}$ mode at $438,449,438$, and $449 \mathrm{~cm}^{-1}$.

Figure 5a shows a transverse dynamic force microscopy image obtained with damping the transverse amplitude of the tip oscillation locked at $30 \%$. The scan represents an area of $1.3 \times 1.3 \mu \mathrm{m}$. This pseudo-topographic image displays an uneven submicronic plateau overhanging a larger flat region. The height of this plateau is about $150 \mathrm{~nm}$. The simultaneous corresponding optical image (Figure 5b) does not show any contrast but only a noised flat image. This last image does not allow distinguishing any optical change in relation with the topographic change. Some examples of section of this TDFM image versus $x$ or $y$ axis show that at the tops of the plateau, either the relief is strongly uneven or the dynamic tip-sample control mechanism does not work very well (Figure 6). Without studying statistically the sample topography, the number of this kind of bumps per $100 \mu \mathrm{m}$ square is between six or eight. The TDFM image shows also that the signal over the plateau is strongly perturbed, while in the valley it becomes very stable during the scan. To understand this anomalous behavior of our device over the plateau, we have recorded the behavior of the vibrating tip versus the sample-tip distance, respectively, for the plateau region and for the valley region (Figure 7). We show only the retract curves to clarify the figure, but in Figure $7 \mathrm{a}$ there is a large hysteresis between the approach and the retract curve, while it is very weak in Figure 7b. This hysteresis in the retract curve is probably caused by the presence of a capillary neck. ${ }^{38}$ The retract curve $b$ displays a continuous curve without any break, the amplitude of the tip's vibration increases with the raise of the tip-sample distance, for example, with the decrease of the force of the interactions between tip and sample. The curves a are not so easy to qualitatively interpret as curve $\mathrm{b}$ and display a weak reproducibility from one probed area over the plateau to another (Figure 7a, curves 1 and 2). This kind of curve will be discussed in the following section with the help of the Raman results.

Indeed, another difference between the plateau and the valley comes from the Raman spectra collected by the tip when it is

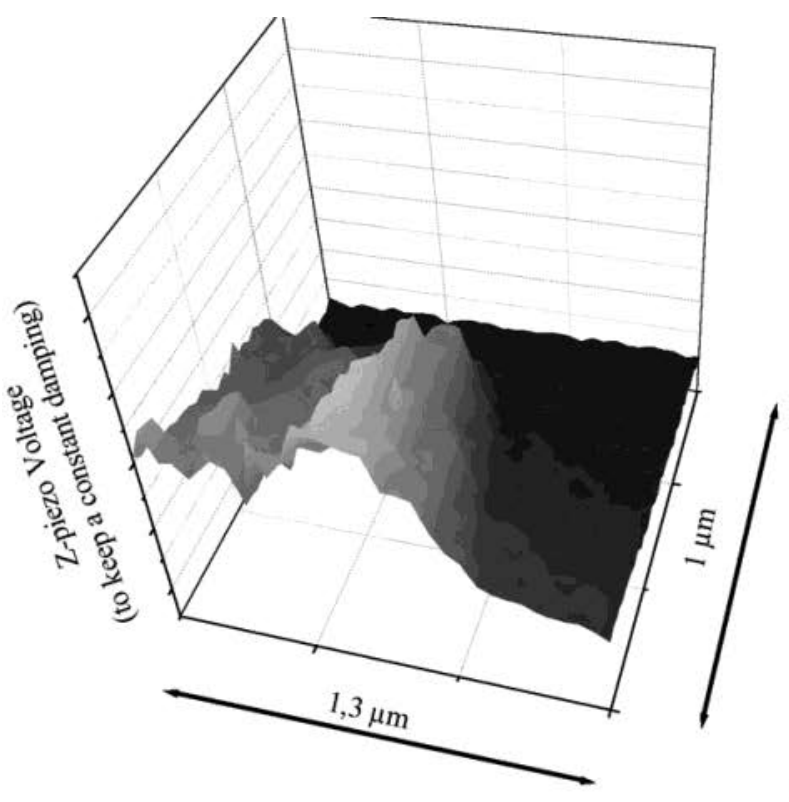

(a)

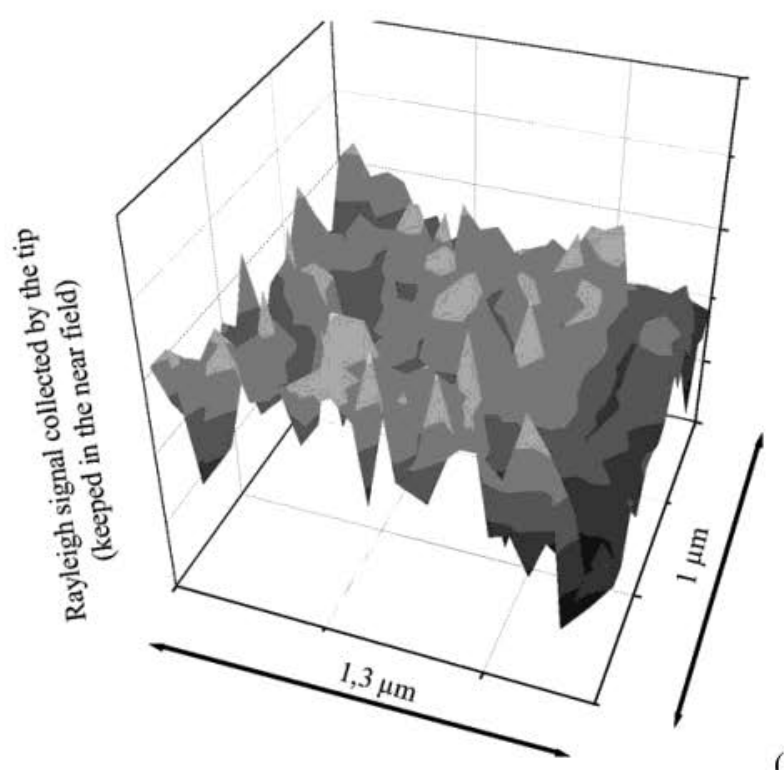

(b)

Figure 5. Images of a small area of the $\mathrm{PbSO}_{4}$ sample $(1.3 \times 1 \mu \mathrm{m})$. At the top (a), an isoforce image; at the bottom (b), the optical near field image of the same area.

maintained close to the surface with the help of the transverse dynamic force feedback loop allowing it to keep a constant value of the damping of the tip's vibration. The spectra from a to $c$ in Figure 8, collected when the tip is kept above the plateau, display a Raman signal clearly shifted by $8 \mathrm{~cm}^{-1}$ forward in comparison with the far field scattered spectrum (Figure 4). The far field wavenumber is, however, retrieved when the tip is moved above the valley (Figure 8d,e). We have verified that, when the tipsurface distance is increased by about $1 \mu \mathrm{m}$, the Raman signal recorded by the tip is strongly decreased (Figure 9). The signal is recovered when the tip is again near to the surface (Figure $9 \mathrm{c})$. Consequently, in the following section, we will assume that the signals collected by the tip are dominated by the inelastic signals of the optical near field.

\section{Discussion}

The previously displayed near field Raman spectra may be interpreted following essentially three ways: 

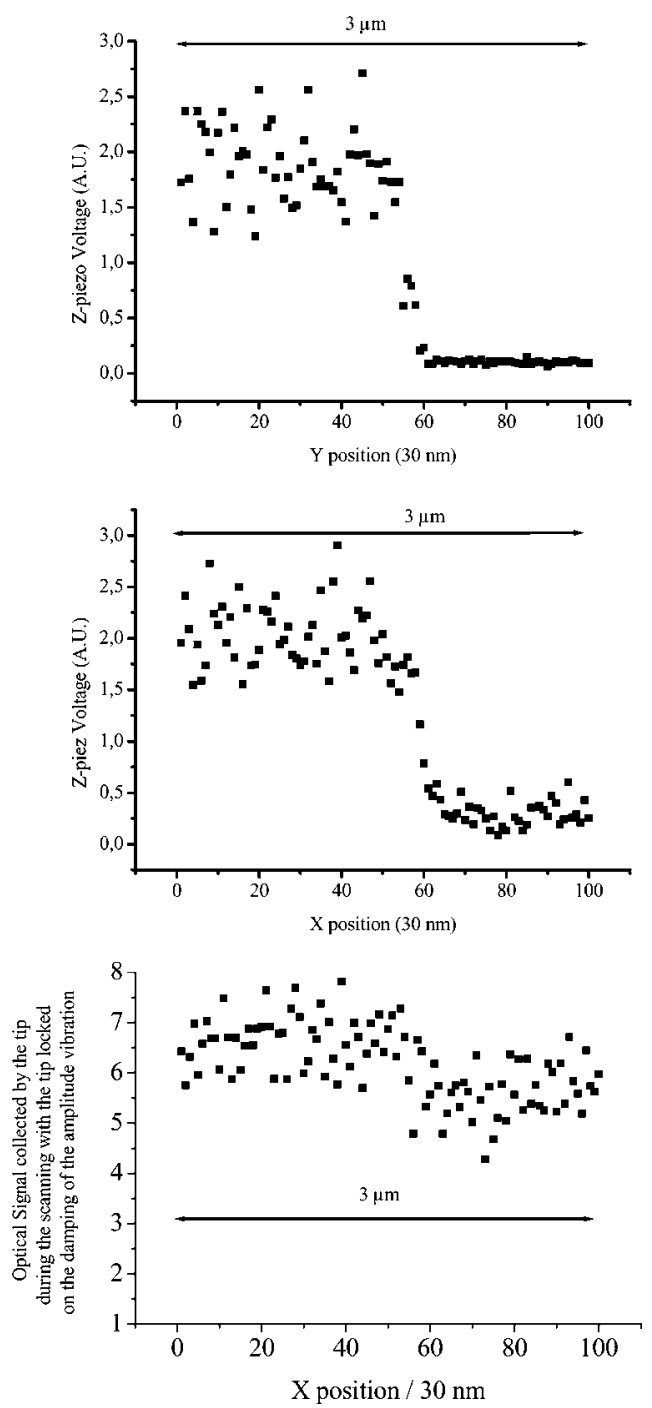

Figure 6. Examples of some sections of the images of Figure 5. Two isoforce section lines and an optical section line.

(i) the inelastic signal obtained in our near field configuration (Figure 1) by the metallic tip is spectrally different from the far field because of the several physical effects, as gradient field, tip effects, nonlinear optical signal, ${ }^{27}$ etc.,

(ii) the tip probes some different crystalline faces with properties characteristic of each face at the interface, and

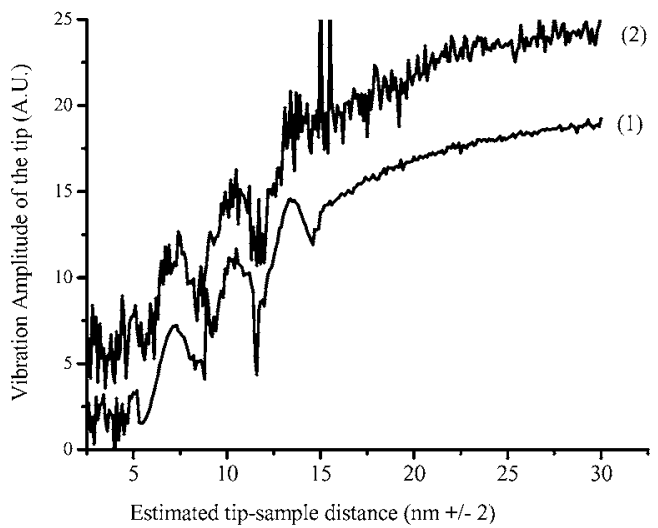

(a) (iii) the collected signals show a heterogeneity of the chemical phases at the surface of our sample, which is not displayed by usual confocal far field measurements (Figure 4).

The first one of the interpretations could be a hypothesis to understand the observed shift of the wavenumber between far field $\left(977 \mathrm{~cm}^{-1}\right)$ and near field $\left(985 \mathrm{~cm}^{-1}\right)$. But it does not explain why the spectra (Figure 8,e) obtained in the valley are different from the spectra collected above the plateau (Figure $8 \mathrm{a}-\mathrm{c})$. This spectral difference, as well as the different retract curves, could be explained by the second possibility, the observation of two kinds of crystalline faces. But no fundamental vibrational argument, coming from the usual interpretation of vibrational modes of the $\mathrm{PbSO}_{4}$ unit cell in the bulk, is able to give an interpretation of this wavenumber shift between two crystalline face types. It is then necessary to assume furthermore that, at the interface, some relaxation process changes the local force field but also changes the vibrational mode wavenumbers. This hypothesis is plausible when we refer to our previous work obtained on a single crystal of silicon. ${ }^{7}$ However, the optical tip probes the surface but also a small volume with a depth of at least $20 \mathrm{~nm}$; consequently, the optical signal should be the sum of surface and volume signal. Thus, in this hypothesis, the collected signal should be composed of two components. Experimentally, only the spectrum in Figure $8 \mathrm{~d}$ is in agreement with this suggestion. The presence of another phase at the surface must be consequently envisaged. Therefore, we have tracked some impurities. Our global chemical analysis proves that the only metallic trace present in our sample is cuprous at an atomic ratio $\mathrm{Cu} / \mathrm{Pb}$ smaller than $5 \times 10^{-4}$. If this weak ratio is kept at the surface, it is not sufficient to explain another superficial phase. We have then checked if the superficial ratio $\mathrm{Cu} / \mathrm{Pb}$ is identical to the global ratio by an X-ray photoelectron spectroscopy measurement. The superficial ratio is then comprised between 0.1 and 0.15 . Since the XPS spectra display only signals of $\mathrm{Pb}(\mathrm{II}), \mathrm{Cu}(\mathrm{II}), \mathrm{Na}(\mathrm{I}), \mathrm{S}(\mathrm{VI}), \mathrm{O}(-\mathrm{II})$, and $\mathrm{C}$ elements, we have recorded the far field Raman spectra of the usual pure cuprous phases. In agreement with the literature (see, i.e., ref 39 ), the closest possibility according to the near field spectra corresponds to the cuprous phase $\mathrm{Cu}\left(\mathrm{SO}_{4}\right) 5 \mathrm{H}_{2} \mathrm{O}$ (Figure 10). ${ }^{39}$

The others phases with three, one, or zero water molecules in the global formula possess, respectively, the symmetric stretching mode of a sulfate group at 1005, and a doublet at 1017 and 1042 or $1052 \mathrm{~cm}^{-1}$. The high water content of the $\mathrm{Cu}\left(\mathrm{SO}_{4}\right), 5 \mathrm{H}_{2} \mathrm{O}$ phase may be an explanation of the particular behavior of the vibrating tip near the surface of this sample.

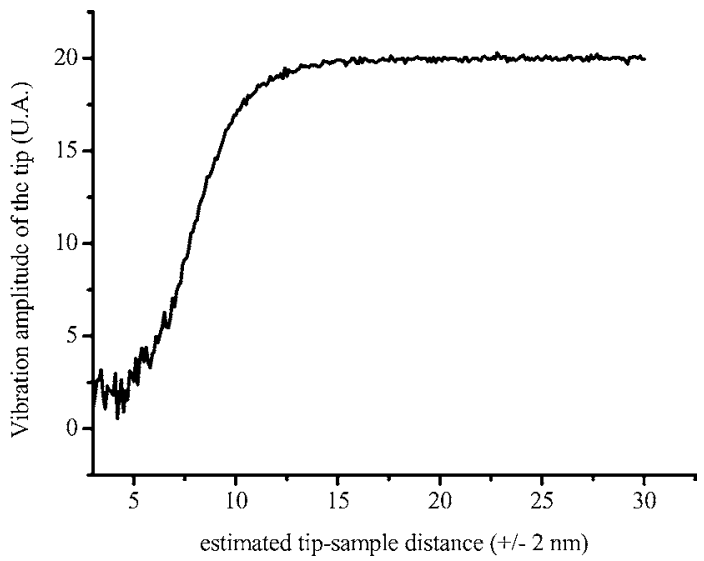

(b)

Figure 7. Two TDFM amplitude-distance curves obtained during a retract curve: at left (a), curves (1) and (2) from different areas over the plateau display the weak reproducibility of this kind of measure and at right (b) over the valley of the image of Figure 5. 


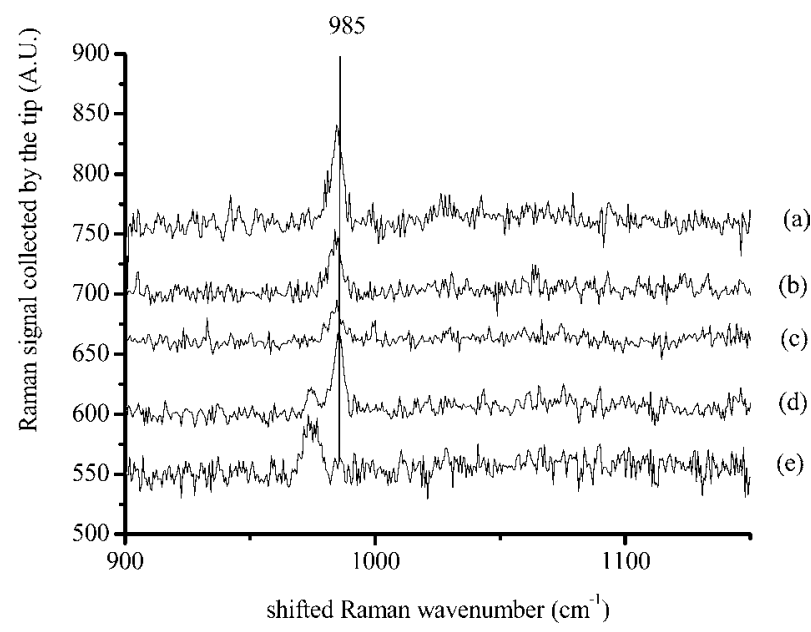

Figure 8. Raman signals collected by the tip during the scan of the sample on the line displayed in Figure 6. The points a-e correspond, respectively, to the $x$ positions of $10(300 \mathrm{~nm}), 25(750 \mathrm{~nm}), 38(1330$ $\mathrm{nm}), 57(2000 \mathrm{~nm})$, and $67(2350 \mathrm{~nm})$ of the first isoforce section of Figure 6. The far field excitation at $514 \mathrm{~nm}$ (power of $1 \mathrm{~mW}$ on the sample) is focused around the tip with the Cassegrain objective on an area of about $100 \mu \mathrm{m}^{2}$. The acquisition time is $120 \mathrm{~s}$.

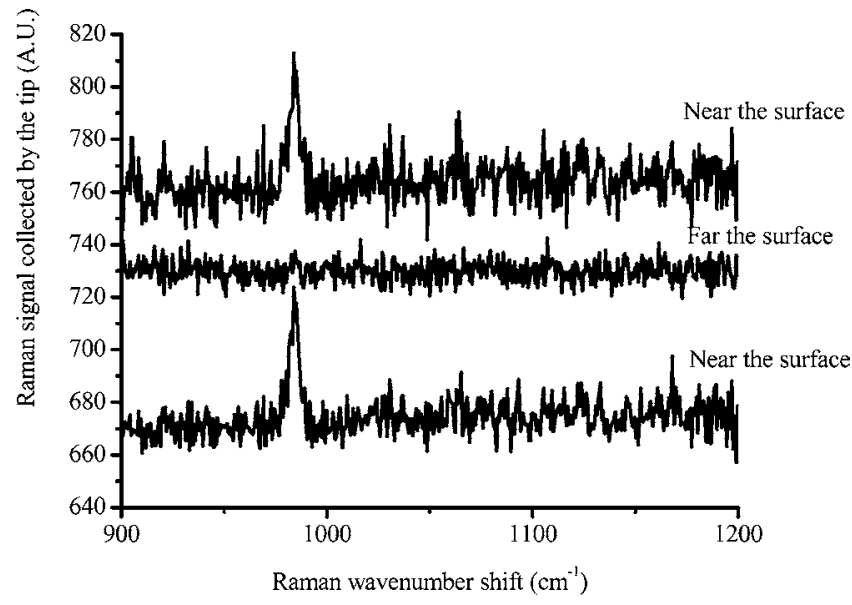

Figure 9. Near field Raman signals collected over the plateau at three sample-tip distances: close to the surface, when the distance feedback loop keeps the tip near the surface (locking vibrating tip amplitude at $50 \%$ of the interaction free amplitude), the top spectrum is obtained before retracting the tip, and the bottom spectrum is collected after a retract-approach cycle, far from the surface; the tip-sample distance is increased from near field position to $700-1000 \mathrm{~nm}$ and maintained at this height during the acquisition of the Raman spectrum. (Acquisition time $120 \mathrm{~s}$, same experimental setup as the one of the experiments of Figure 8.)

Indeed, the water molecules, present at the surface, constitute preferential sites for the adsorption of other water molecules at the equilibrium in the vapor phase, as the first adsorbed molecule on the silica surface. ${ }^{40}$ Thus, when the tip probes this kind of chemical heterogeneity, the tip-sample interaction changes strongly: over the cuprous phase, the interactions are dominated by capillary forces with viscous effects, ${ }^{38}$ while over the lead phase, the interactions are rather dominated by usual effects as dipolar-dipolar or van der Waals interactions. This vision is in agreement with the TDFM amplitude distance curves recorded in Figure 7. Indeed, these curves may be interpreted as James et al. have explained their curves obtained with $\mathrm{Nafion}^{+}$and $\mathrm{Cs}^{+}$samples. Our curve obtained over the valley (Figure $7 \mathrm{~b}$ ) is near the TDFM curve obtained at $10 \%$ humidity with Nafion $\mathrm{Cs}^{+}$, while the curve in Figure $7 \mathrm{a}$ is near the curve obtained with Nafion $\mathrm{H}^{+}$at $10 \%$ of humidity. These differences may

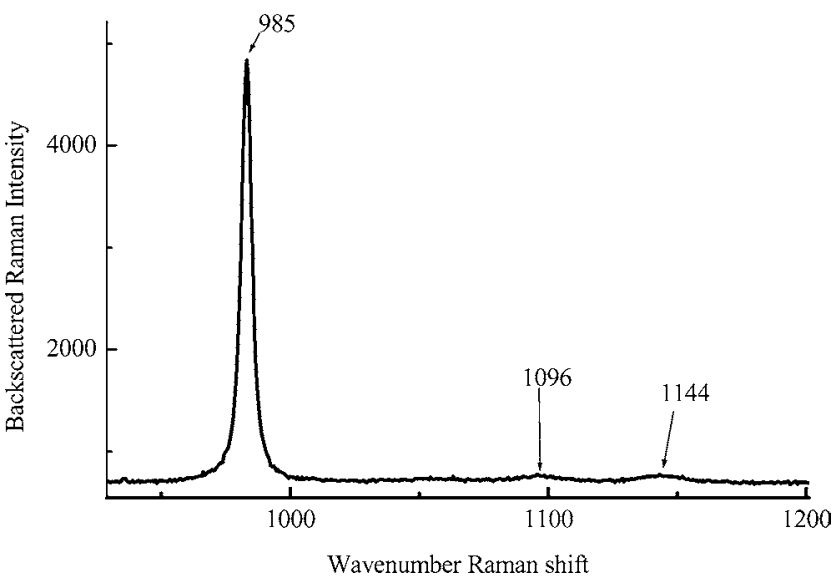

Figure 10. Far field usual backscattered Raman spectrum of $\mathrm{CuSO}_{4}$, $5 \mathrm{H}_{2} \mathrm{O}$ obtained with an excitation at $514 \mathrm{~nm}$ and a power of $10 \mathrm{~mW}$ focused with an objective $\times 50$ (numerical aperture of 0.5 ).

show a mixed hydrophobic/hydrophilic behavior with a much larger adhesive force over the water-rich regions, in agreement with our chemical local analysis obtained by the NFR spectroscopy. Our homemade device is not yet able to record a complete real time frequency TDFM spectrum of the vibrating tip simultaneously with our NFR measurements. Consequently, we are not able now to evaluate the elastic and dissipative components of vibrating movements of the tip versus the spatial region probed. Indeed, the information provided by the retractapproach curve alone at a fixed frequency of the vibrating tip is insufficient to determine the kind of probe-sample interaction since two ways of amplitude decrease are possible:

(i) if the dissipative force remains constant while the elastic component increases and

(ii) if the variation of the dissipative component dominates the change of the elastic component.

However, even if the TDFM spectroscopy is necessary, it is not sufficient if used alone, to understand the physical chemistry of the interfaces. Therefore, we think that this work shows a new experimental complementary approach. Indeed, this paper demonstrates the possibilities of our device to record simultaneously at the same submicronic scale the chemical spectral fingerprint and the interactions between tip and sample surface. Thus, the near field Raman spectroscopy coupled with the TDFM spectroscopy should become a unique tool to give a complete description of the chemistry at heterogeneous liquidsolid interfaces, by combining submicronic chemical structure investigation with nanoscale force measurements.

\section{Conclusions}

This paper demonstrates the possibilities of the near field Raman spectroscopy (NFRS) on a $\mathrm{PbSO}_{4}$ sample, which does not present any resonance or optical enhancement effects. The Raman spectrum of a submicronic particle is obtained by our NFR device, while the confocal Raman microscope is not able to distinguish it. This NFR signal decreases strongly with the tip-surface distance and displays some spectral changes with a lateral scan of about $200 \mathrm{~nm}$. These spectral data of the optical near field are concomitantly recorded with some force measurements obtained by the transverse dynamic force microscope, of which the first use is to maintain the optical tip near the surface. Thus, the chemical differences are identified by the submicronic NFR spectra and by the probe-adhesion measurements. On the sample analyzed here, the inhomogeneous distribution of surface water displayed by the amplitude tipsample distance curves is explained by the presence of 
nanophases of $\mathrm{CuSO}_{4}, 5 \mathrm{H}_{2} \mathrm{O}$ characterized by the NFR spectra. Future improvement of our device will consist of recording the real-time vibrating tip frequency spectrum to separate the two effects of changes in resonance frequency and oscillation damping. Thus, one could correlate, with certitude, chemical identification and tip-surface interactions at the interface. In our future papers, we will also develop the submicronic spectroscopy approach by using molecular probes to investigate the inhomogeneous reactivity often assumed at the interfaces of oxide or hydroxide mineral particles. ${ }^{41}$

Acknowledgment. The authors want acknowledge Mr. G. Antoine for his help in electronic conceptions, Mr. M. Spajer for his competencies in optical near field, and Mr. J. Lambert for his XPS analyses.

\section{References and Notes}

(1) Eggleston, C. M.; Hug, S.; Stumm, W.; Sulzberger, B.; Dos Santos Afonso, M. Geochim. Cosmochim. Acta 1998, 62, 585-593.

(2) Stack, A. G.; Higgins, S. R. Eggleston, C. M. Geochim. Cosmochim. Acta 2001, 65, 3055-3063.

(3) Grausem, J.; Humbert, B.; Burneau, A.; Oswalt, J. Appl. Phys. Lett. 1997, 70, 1671-1674.

(4) Grausem, J. Thesis. Nancy 1998

(5) Grausem, J.; Davy, S.; Humbert, B.; Spajer, M.; Courjon, D. Submicronic Raman experiments using a near field scanning microscope 24th Annual Conference of the Federation of Analytical Chemistry and Spectroscopy Societies (FACSS); Rhode Island, October 1997.

(6) Humbert, B.; Grausem, J.; Spajer, M.; Courjon, D. Chim. Nouvelle 1999, 17,1 .

(7) Grausem, J.; Humbert, B.; Spajer, M.; Courjon, D.; Burneau, A.; Oswalt, J. J. Raman Spectrosc. 1999, 30, 833-840.

(8) Jahncke, C. L.; Paesler, M. A.; Hallen, H. D. Appl. Phys. Lett. 1995, 67, 2483.

(9) Jahncke, C. L.; Hallen, H. D.; Paesler, M. A. J. Raman Spectrosc. 1996, 27,579

(10) Synge, E. H. Philos. Mag. 1928, 6, 356

(11) Synge, E. H. Philos. Mag. 1932, 13, 297.

(12) Binnig, G.; Rohrer, H.; Weibel, E. Phys. Rev. Lett. 1982, 49, 57.

(13) Pohl, D. W.; Denk, W.; Lanz, M. Appl. Phys. Lett. 1984, 44, 651.

(14) Toledo-Crow, R.; Yang, P. C.; Chen, Y.; Vaez-Iravani, M. Appl. Phys. Lett. 1992, 60, 2957. 2484.
(16) Betzig, E.; Trautman, J. Science 1992, 257, 189.

(17) Webster, S.; Batchelder, D. N.; Smith, D. A. Appl. Phys. Lett. 1998, 72,1478 .

(18) Tsai, D. P.; Othonos, A.; Moskovits, M. Appl. Phys. Lett. 1994, 64, 1768.

(19) Smith, D. A.; Webster, S.; Ayad, M.; Evans, S. D.; Fogherty, D.; Batchelder, D. Ultramicroscopy 1995, 61, 247.

(20) Sharp, S. L.; Warmack, R. J.; Goudonnet, J. P.; Lee, I.; Ferrell, T. L. Acc. Chem. Res. 1993, $26,377$.

(21) Emory, S. R.; Nie, S. Anal. Chem. 1997, 69, 2631.

(22) Zeisel, D.; Deckert, V.; Zenobi, R.; Vo-Dinh, D. T. Chem. Phys. Lett. 1998, 283, 381 .

(23) Webster, S.; Batchelder, D. N.; Smith, D. A. Appl. Phys. Lett. 1998, 72,1478 .

(24) Webster, S.; Smith, D. A.; Batchelder, D. N. Spectrosc. Eur. 1998, $10,22$.

(25) Narita, Y.; Tadokoro, T.; Ikeda, T.; Saiki, T.; Mononobe, S.; Ohtsu, M. Appl. Spectrosc. 1998, 52, 1141.

(26) Ayars, E. J.; Hallen, H. D. Appl. Phys. Lett. 2000, 76, 3911.

(27) Ayars, E. J.; Hallen, H. D.; Jahncke, C. L. Phys. Rev. Lett. 2000 85,4180 .

(28) Ayars, E. J.; Jahncke, C. L.; Paesler, M. A.; Hallen, H. D. J. Microsc. 2001, 202, 142.

(29) Hayazawa, N.; Inouye, Y.; Sekkat, Z.; Kawata, S. Chem. Phys. Lett. 2001, 335, 369 .

(30) Sands, H. S.; Demangeot, F.; Bonera, E.; Webster, S.; Bennett, R.; Hayward, I. P.; Marchi, F.; Smith, D. A.; Batchelder, D. N. J. Raman Spectrosc. 2002, 33, 730

(31) Hayazawa, N.; Tarun, A.; Inouye, Y.; Kawata, S. J. Appl. Phys. 2002, 92, 6983 .

(32) Hayazawa, N.; Yano, T.; Watanbe, H.; Inouye, Y.; Kawata, S. Chem. Phys. Lett. 2003, 376, 174.

(33) Gucciardi, P. G.; Trusso, S:, Vasi, C.; Patane, S.; Allegrini, M. Appl. Optics 2003, 42, 2724.

(34) Hartschuh, A.; Sanchez, E. J.; Xie, X. S.; Novotny, L. Phys. Rev. Lett. 2003, 90, 1 .

23

(35) Courjon, D.; Sarayedine, K.; Spajer, M. Opt. Commun. 1989, 71,

(36) Paesler, M. A.; Moyer, P. J. Near Field Optics, Theory, Instrumentation and Applications; J. Wiley and Sons: New York, 1996.

(37) Pohl, D. W.; Courjon, D. Near field Optics NATO Series 242; Kluwer Academic: Dordrecht, The Netherlands, 1993.

(38) James, P. J.; Antognozzi, M.; Tamayo, J.; McMaster, T. J.; Newton, J. M.; Miles, M. J. Langmuir 2001, 17, 349.

(39) Chang, H.; Huang, P. J. J. Chin. Chem. Sol. 1998, 45, 59.

(40) Burneau, A.; Humbert, B.; Gallas, J.; Lavalley, J. ACS Adv. Chem. Ser. 1994, 234, 199.

(41) Phambu, N.; Humbert, B.; Burneau, A. Langmuir 2000, 16, 6200. 